\title{
Mobile Assessment System for Shoulder Joint Rehabilitation: System Development and Preliminary Study
}

\author{
Yoon Sang Kim ${ }^{1}$, Seong-wook Jang ${ }^{1}$ and Yon-Sik Yoo ${ }^{2}$ \\ ${ }^{1}$ Department of Computer and Science Engineering, Korea University of Technology \\ and Education, Chunan, Chungnam, South Korea \\ ${ }^{2}$ Department of Orthopedic Surgery, Hallym University Medical Center, Dongtan, \\ Gyunggido, South Korea \\ yoonsang@koreatech.ac.kr
}

\begin{abstract}
This paper proposes the mobile assessment system for shoulder joint rehabilitation. To monitor the patient's condition during the rehabilitation process, it is necessary to assess the initial condition and monitor the progress before and after the treatment. For the assessment, the shoulder joint angle in certain positions needs to be measured. The proposed system measures and assesses the patient's joint angles in four different positions using smart sensors embedded in the smartphones such as the accelerometer and the gyroscope. The performance of the proposed system is evaluated by comparing the assessment results with measurements using the goniometer.
\end{abstract}

Keywords: Smartphone application, Shoulder joint, Rehabilitation, Mobile assessment, Sensor measurement

\section{Introduction}

The medical industry comprising of medical devices and services is expanding due to the rapid progress of information technology. Information technology is being applied to all kinds of medical devices, ranging from expensive surgical robots to less expensive small medical devices. In addition, the convergence of IT and medicine has enabled the digitization of medical data, enhancing the credibility of diagnoses and tests prescribed by doctors. Furthermore, medical services have become more efficient with the emergence of cuttingedge technology medical devices which can diagnose, test, and perform surgery with a single equipment.

The primary function of medical devices is to measure biological data using sensors, which makes sensor technology a major factor in medical devices merged with IT. Various sensors are being used in the medical system for diagnosis and examination and more recently, the research and development of various smart sensor-based medical systems for diagnosis and examination are underway. The aforementioned smart sensors refer to intelligent sensors which are produced with MEMS and SoC (system on chip) technology, delivering high-level functions and high-precision performance. Smartphones carry smart inertial sensors that can measure angular acceleration, which are evolving into motion sensors and IMU sensors by combining the three-axis acceleration sensor with the three-axis gyro sensor. In addition, smartphones are gaining attention as the next generation biological signal measurement equipment through their various high-performance sensors such as high-sensitivity mikes and high-resolution cameras. 
Meanwhile, safety accidents and extreme exercises have been contributing to a steady increase in the number of patients with functional disorders. Continuous rehabilitation is required for such patients. Doctors record the initial state of the patient and monitor any changes prior to and following the treatment by measuring the joint angle. Therefore, an accurate measurement of the joint movement in motion is a critical matter. The joint angle measurement using electrogoniometer, however, has a drawback of lowering the level of accuracy due to the abrasion of internal resistance caused by repeated use [2-3]. To counter such problems, the study was conducted using attachable sensors such as fiber-optic angle sensor, optical fiber sensor, and internal measurement unit (IMU) to measure the joint angles [4-7]. Attachable sensors increase the accuracy of the joint angle measurement data by attaching the sensor to the body but it lacks convenience [8-9].

This paper proposes the measurement and evaluation system for shoulder joint rehabilitation using smartphones. In order to check for any changes in a patient's condition over the course of rehabilitation, joint angles in specific positions needs to be measured. The proposed system measures and evaluates the patient's shoulder joint angles in 4 different positions using the smart sensors (accelerometer and gyroscope) embedded in smartphones. The performance of the proposed system is evaluated by comparing the assessment results with measurements using the goniometer.

\section{The Angle Measurement for the Evaluation of Shoulder Joint Rehabilitation using Smartphones}

The proposed system measures the shoulder joint angle to evaluate the rehabilitation using accelerometer and gyroscope embedded in the smartphones. The measurement using the proposed system has three steps as shown in Figure 1: 1) Initialize the smartphone to offset the coordinate in the accelerometer with the radius bone. 2) Make shoulder joint rehabilitation motion with the smartphone attached. 3) Measure the shoulder joint angle holding the final motion.

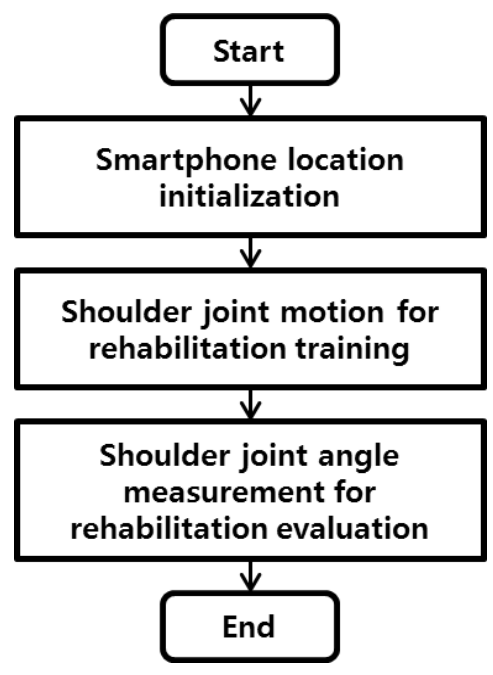

Figure 1. The measurement procedure for the evaluation of shoulder joint rehabilitation using a smartphone

In order to measure the shoulder joint angle, the accelerometer has to be aligned with the coordinate system of the shoulder joint. Figure 2(a) shows the three-dimensional coordinates 
of the accelerometer embedded in the smartphone; the $\mathrm{x}$-axis plots horizontal movements with the device facing the front, the y-axis plots vertical movements, and the z-axis plots back and forth movements of the device itself. Therefore, the initialization is performed to register the accelerometer and coordinates of the radius bone as shown in Figure 2(b).

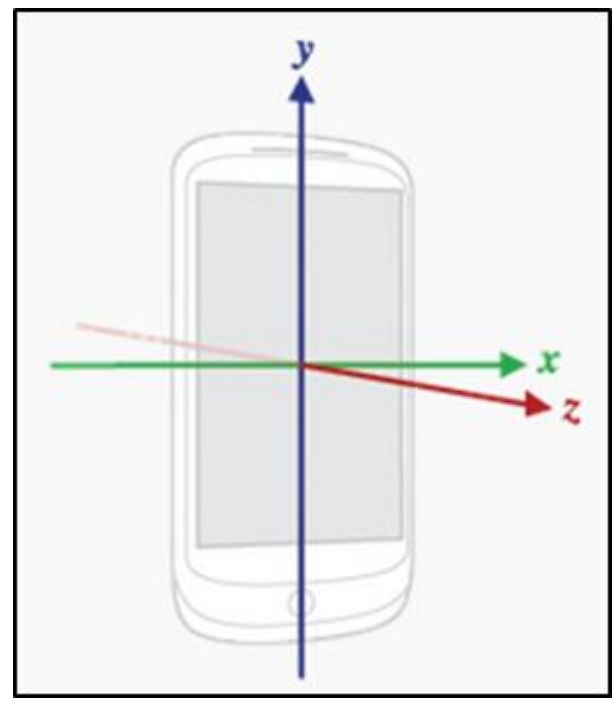

(a) Coordinates of accelerometer in the smartphone

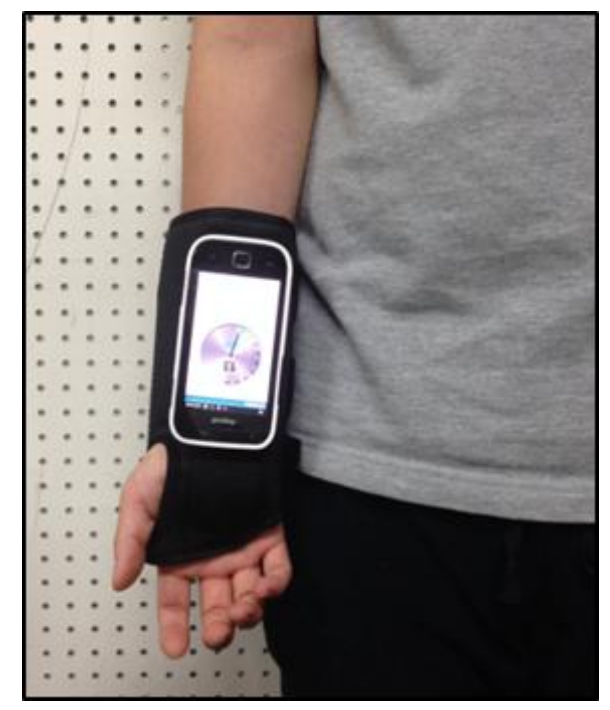

(b) Registration of the accelerometer sensor and radius bone coordinate system

Figure 2. The location initialization of Smartphone

The accelerometer in the smartphone estimates the rotation displacement using the gravity acceleration [10]. Because rotation displacement estimation using gravity acceleration carries intrinsic measurement errors due to the gravity factor, the proposed system separates the gravity component using the low pass filter as shown in Equation (1) [11].

$$
g_{\mathrm{i}}(k)=\alpha g_{\mathrm{i}}(k-1)+(1-\alpha) a_{\mathrm{i}}(k)
$$

where, $g_{\mathrm{i}}(k)$ is gravity component, $a_{\mathrm{i}}(k)$ is the acceleration of axis-I, I is axis $\mathrm{x}, \mathrm{y}, \mathrm{z}$ value respectively.

The acceleration of the smartphone is obtained by deducting the gravity component of three axis found in the equation (1) from the acceleration found in the equation (2).

$$
\overline{a_{\mathrm{i}}}(k)=a_{\mathrm{i}}(k)-g_{\mathrm{i}}(k)
$$

Based on the equation (1) and (2), the smartphone application is implemented for the measurement of the shoulder joint angle. Android provides API creating the event if the current sensor value changes in the comparison with the previous sensor value measured periodically according to a set schedule. The implemented application measures the angle by applying then low pass filter to the accelerometer and gyroscope sensor data obtained from API. The angle measurement application GUI for rehabilitation evaluation implemented in the smartphones is illustrated in Figure 3. 


\section{VirtualClinic}

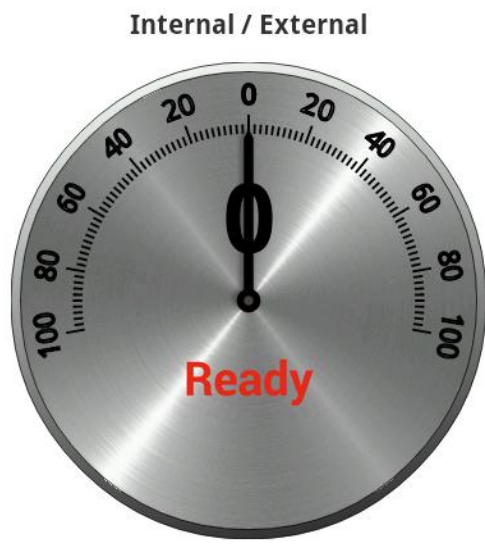

(a) Before the measuring

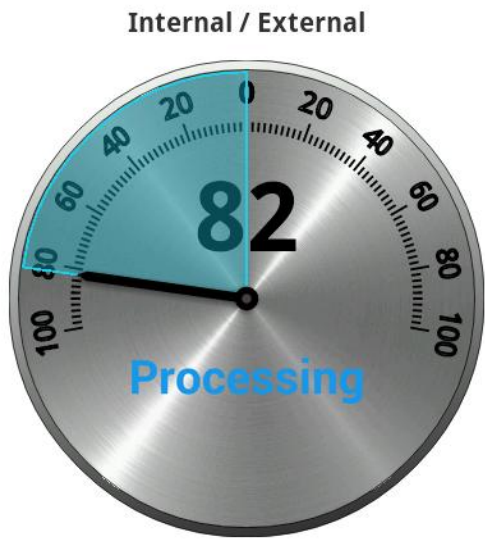

(b) During the measuring

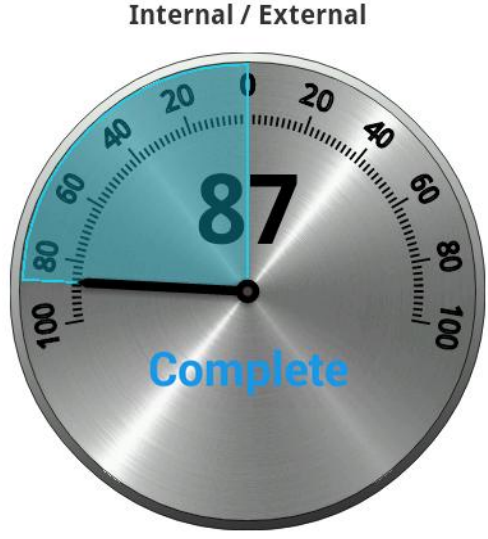

(c) After the measuring

Figure 3. Angle measurement application (GUI) implemented for the shoulder joint rehabilitation evaluation

The measurement is conducted based the following three steps: 1) "Ready state"(Figure 3a) is the step for adjusting basic posture of patient before measurement. 2) "Process state"(Figure $3 b$ ) is the step for measuring angle. In this step, measuring ROM (Range of Motion) covers from 0 degree to 180 degree. ABD and FF motion measure angle of vertical motion. IR and ER motion measure angle of horizon rotation on fixed upper arm. 3) "Complete state" (Figure 3c) is the final step.

\section{The Preliminary Study and Discussion}

As a preliminary experiment for the clinical study, this section deals with the evaluation of proposed shoulder joint rehabilitation system by comparing the measurement result using 
goniometer with the proposed system. The performance is evaluated in a way that the measurement error of the system is calculated based on the measured result using goniometer. During the experiment, the measurement of the shoulder joints is carried out in four motions used for the rehabilitation evaluation; abduction, forward flexion, internal rotation, external rotation [12]. Figure 4-7 illustrates the process of the measurement and evaluation for the rehabilitation using the smartphones. The abduction (ABD) movement refers to the arm being lifted as high as possible sideways; forward flexion (FF) is the movement raising an arm towards the front; internal rotation (IR) and external rotation (ER) is when elbows are rotated to their fullest extent both inwards and outwards while being fixed to the sides. The experiment was conducted on five subjects.

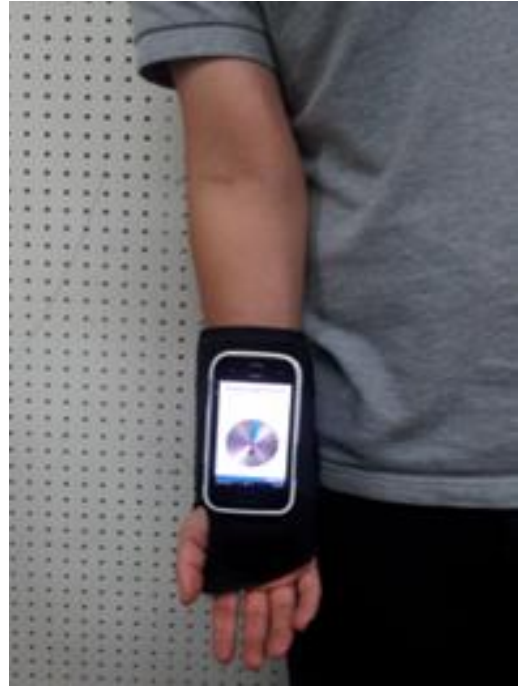

(a) ABD initial position

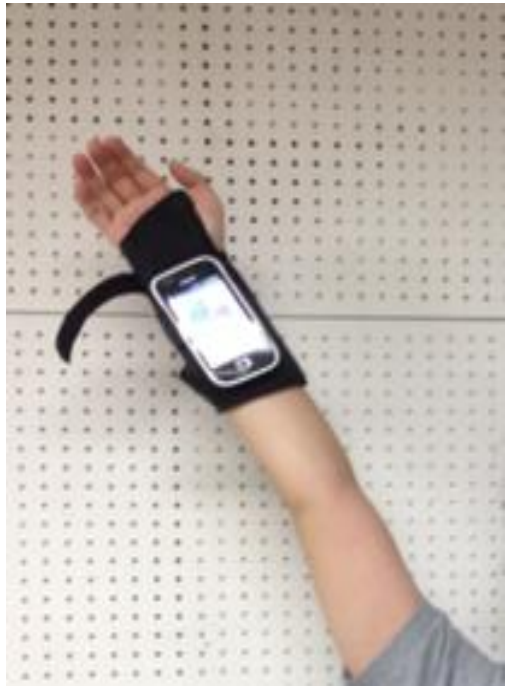

(c) ABD final position

Figure 4. ABD for the rehabilitation

$\mathrm{ABD}$ is a motion which one moves arm toward the outside of the body in the frontal plane. Maximum range of $\mathrm{ABD}$ motion is approximately up to 180 degree. This movement occurs more frequently in special exercise and physical training than daily activity. ABD measurement is performed as shown in Figure 4. Initial position (Figure 4a) is maintaining the position to make arm down until device gets perpendicular to the ground. Final position (Figure 4b) is measured on the position when one can lift one's arm up to as high as possible in initial position. 


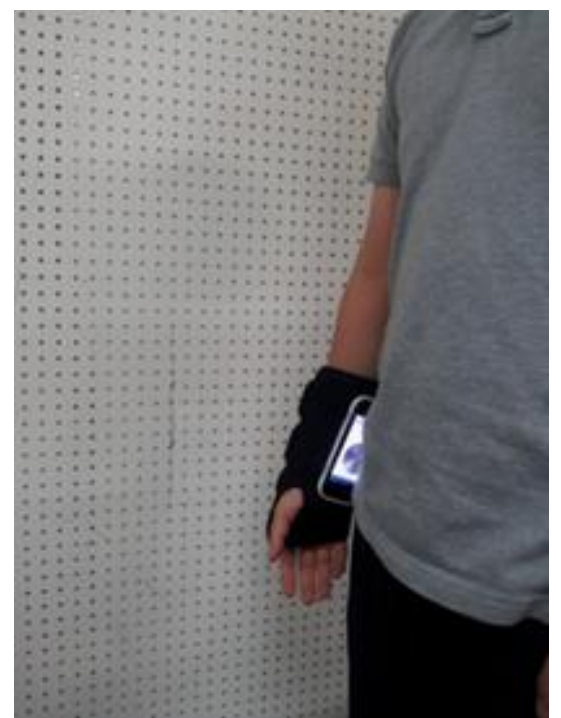

(a) FF initial position

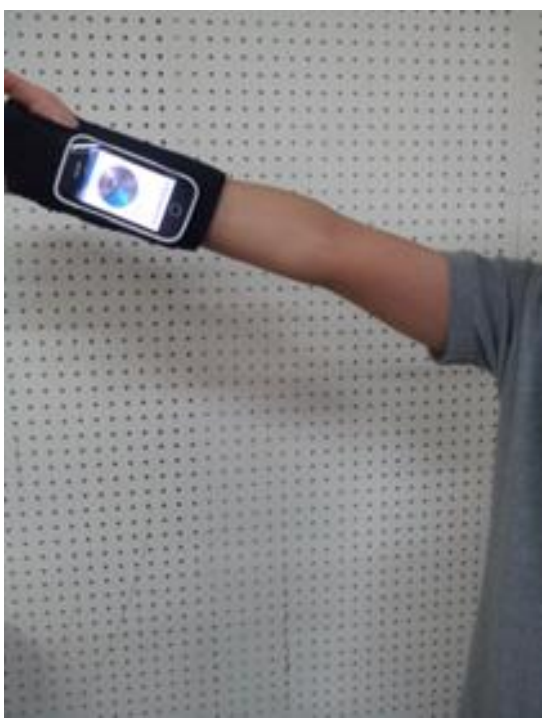

(b) FF final position

\section{Figure 5. FF for the rehabilitation}

FF movement is a motion which one lifts up arm to the front of the body in the sagittal plane. The maximum degree of ROM in this movement reaches up to 180 degree. The most common shoulder motion occurs as the small bending range when one types on a keyboard, presses a button, or manipulating a steering wheel. FF measurement is achieved as shown in Figure5. Initial position (Figure 5a) is maintaining the position to make arm up until device gets perpendicular to the ground. Final position (Figure 5b) is measured by lifting arm up vertically in fixed scapular.

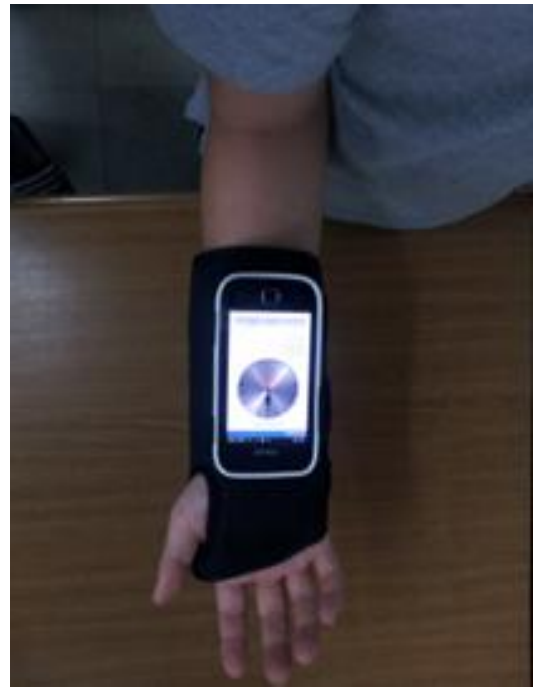

(a) IR, ER initial position

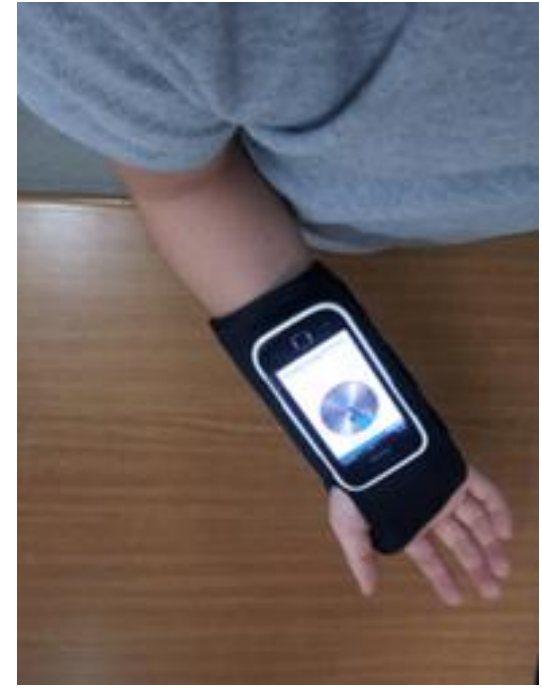

(b) IR final position

Figure 6. The internal rotation(IR) for the rehabilitation 
The internal rotation movement is the movement when one moves his arm as a spiral. Ordinary ROM is approximately 30 degree and sometimes it is coupled to the bending motion such as the motion for grabbing a ball. The internal rotation measurement is achieved while holding the elbow to side (as shown in Figure 6a) and pulling to the inside of body as much as possible (Figure 6b).

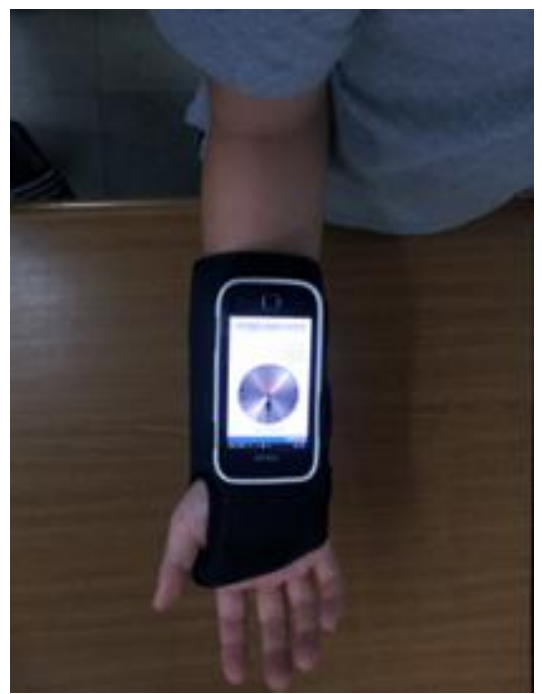

(a) IR, ER initial position

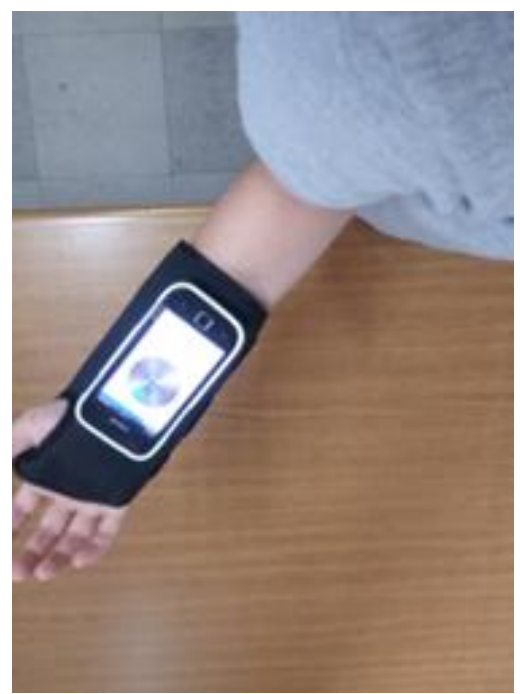

(b) ER final position Figure 7. The external rotation(ER) for the rehabilitation

The external rotation movement is a motion which arm is rotating outwardly from body and it is not common in everyday movement. The external rotation measurement is measured after holding elbow (Figure 7a) to side and pulling to the outside of body as much as possible (Figure 7b). The measurement results using the conventional method with goniometer and the proposed ones with the smartphone are illustrated in the Table 1. ROM results measured by conventional method were as follows: measurement results on ABD motion were 156(case 1), 163(case 2), 154(case 3), 153(case 4), and 142(case 5); measurement results on FF motion were 92(case 1), 101(case 2), 87(case 3), 94(case 4), and 94(case 5); measurement results on IR motion were 27(case 1), 32(case 2), 77(case 3), 31(case 4), and 53(case 5); measurement results on ER motion were 31(case 1), 51(case 2), 36(case 3), 36(case 4), and 39(case 5). Next, ROM results measured by the proposed method were as follows: measurement results on ABD motion were 157(case 1), 167(case 2), 156(case 3), 154(case 4), and 143(case 5); measurement results on FF motion were 95(case 1), 99(case 2), 91(case 3), 93(case 4), and 98(case 5); measurement results on IR motion were 29(case 1), 33(case 2), 82(case 3), 32(case 4), and 59(case 5); measurement results on ER motion were 29(case 1), 51(case 2), 38(case 3), 34(case 4), and 42(case 5). The measurement result are as follows: ADB showed the angular displacement from $142^{\circ}$ to $167^{\circ}$; FF showed the angular displacement from $87^{\circ}$ to $101^{\circ}$; IR and ER showed the angular displacements of $27-82^{\circ}$ and $29-51^{\circ}$ respectively. The error ranged from $0^{\circ}$ to $6^{\circ}$ and the mean error was $2.9375^{\circ}$. 
Table 1. The results of shoulder ROM (degree)

\begin{tabular}{|c|c|c|c|c|c|}
\hline \multicolumn{2}{|c|}{ Specimen } & $\mathrm{ABD}$ & $\mathrm{FF}$ & IR & $E R$ \\
\hline \multirow{3}{*}{ Case 1.} & Conventional method & 156 & 92 & 27 & 31 \\
\hline & Proposed method & 157 & 95 & 29 & 29 \\
\hline & Error rate & -1 & -3 & -2 & 2 \\
\hline \multirow{3}{*}{ Case 2.} & Conventional method & 163 & 101 & 32 & 51 \\
\hline & Proposed method & 167 & 99 & 33 & 51 \\
\hline & Error rate & -4 & 2 & -1 & 0 \\
\hline \multirow{3}{*}{ Case 3.} & Conventional method & 154 & 87 & 77 & 36 \\
\hline & Proposed method & 156 & 91 & 82 & 38 \\
\hline & Error rate & -2 & -4 & -5 & -2 \\
\hline \multirow{3}{*}{ Case 4.} & Conventional method & 153 & 94 & 31 & 36 \\
\hline & Proposed method & 154 & 93 & 32 & 34 \\
\hline & Error rate & -1 & 1 & -1 & 2 \\
\hline \multirow{3}{*}{ Case 5.} & Conventional method & 142 & 94 & 53 & 39 \\
\hline & Proposed method & 143 & 98 & 59 & 42 \\
\hline & Error rate & -1 & -4 & -6 & -3 \\
\hline
\end{tabular}

Conventional method: Goniormeter/Proposed method: Smartphone

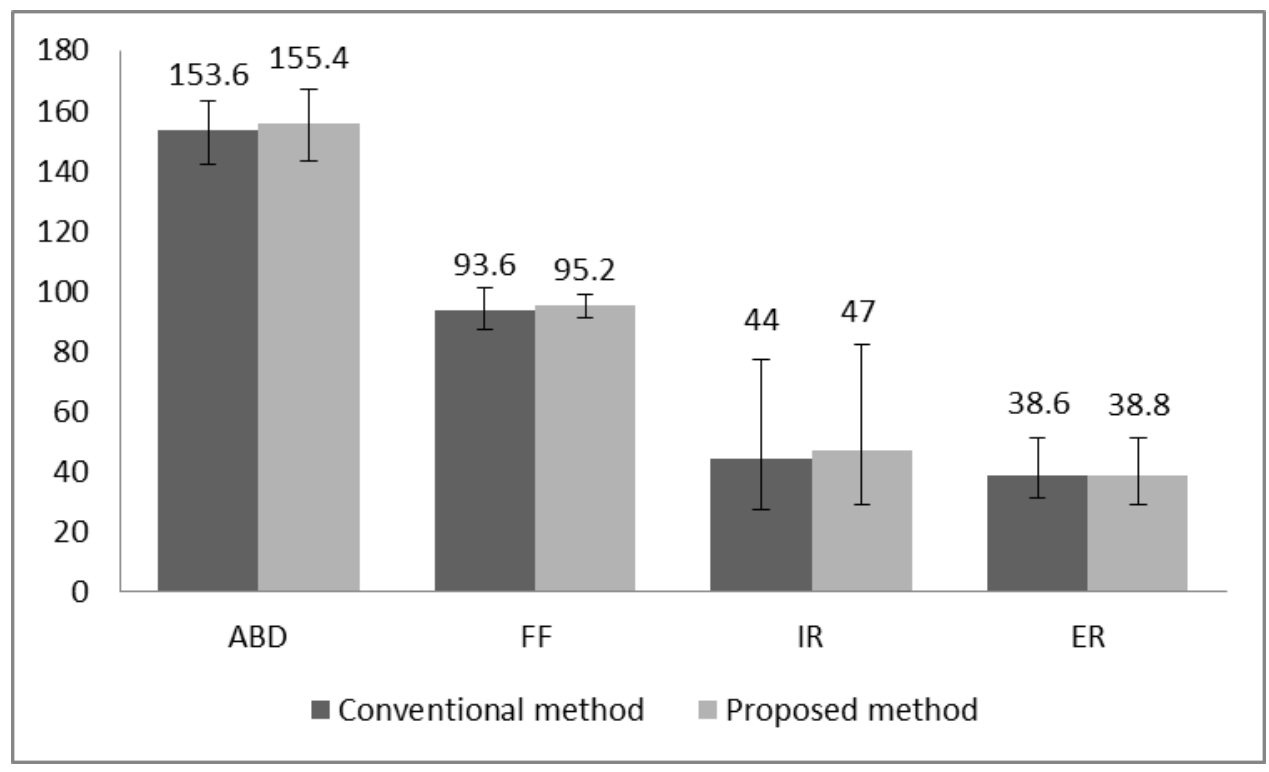

Figure 8. The average results of shoulder ROM (degree) 
Figure 8 shows the mean values based on Table 1 . The mean error between the proposed method and the conventional method was $1.65^{\circ}$.

\section{Conclusion}

In this paper, the angle measurement system for the rehabilitation evaluation using the smartphone has been discussed. Based on the accelerometer and gyroscope embedded in the smartphone, the proposed system measured the patient's shoulder joint angle in four different positions. The performance of the system was reviewed by comparing the measurement results of the method using goniometer with the proposed one which was conducted on five subjects. The experiment result verified that the measurement result of the system, unlike its counterpart using goniometer, was within the allowable range of error (The mean error was approximately 2.9375), which validated the viability of the proposed system. While the proposed system must remain parallel with the radius bone at all times for measurement accuracy, the wristband provides relatively accurate measurements of the shoulder joint angle from simple movements by securing the device. The shoulder joint rehabilitation assessment system presented in this paper is expected to be implemented in blind tests for patients with various shoulder joints disorders in the future.

\section{Acknowledgements}

This research was supported by Basic Science Research Program through the National Research Foundation of Korea (NRF) funded by the Ministry of Education (2013R1A1A2011589).

\section{References}

[1] Y. S. Kim, K. U. Kyung and S. T. Park, "Smart Sensor: Transparent and Flexible Pressure Sensor", Electronics and Telecommunications Trends, ETRI, (2012), pp. 64-72.

[2] http://www.emgsrus.com/prod_access_goniometers.html.

[3] http://www.ergotest.com/products/Goniometer/Goniometer.html.

[4] S. G. Kim, S. H. Shin, D. Jeon, S. H. Hong, H. I. Sim, K. W. Jang, W. J. Yoo and B. Lee, "Fiber-optic Goniometer to Measure Knee Joint Angle for the Diagnosis of Gait Disturbance", The Transactions of the Korean Institute of Electrical Engineers, vol. 62, no. 7, (2013).

[5] G.-I. Jung, J. -S. Kim, D. -H. Hur, H. -D Yu, S. -H. Lim, J. -H. Choi, J. -H. Lee, G. -M. Eom and J. -H, Jun, "Angular Displacement Measurement Using Optical Sensor", The Transactions of the Korean Institute of Electrical Engineers, vol. 60, no. 10, (2011).

[6] J. Favre, R. Aissaoui, B. M. Jolles, J. A. de Guise and K. Aminian, "Functional calibration procedure for 3D knee joint angle description using inertial sensors", Journal of biomechanics, vol. 42, no. 14, (2009).

[7] S. Bakhshi, M. H. Mahoor and B. S. Davidson, "Development of a body joint angle measurement system using IMU sensors", Engineering in Medicine and Biology Society, EMBC, 2011 Annual International Conference of the IEEE. IEEE, (2011).

[8] X. Long, B. Yin and R. M. Aarts, "Single-Accelerometer-Based Daily Physical Activity Classification", Proceedings of IEEE Engineering in Medicine and Biology Society, (2009).

[9] L. Bao and S. S. Intille, "Activity Recognition from User-Annotated Acceleration Data", Proceedings of the International Conference on Pervasive Computing, Lecture Notes in Computer Science, (2004).

[10] M. Pedley, "Tilt sensing using a three-axis accelerometer", Freescale Semiconductor, Inc., (2013).

[11] http://developer.android.com/reference/android/hardwarre/sensorevext.thml.

[12] Norkin, C. Cynthia and D. J. White, "Measurement Of Joint Motion: A Guide To Goniometry", FA Davis Company, Philaadelphia, (2009). 


\section{Authors}

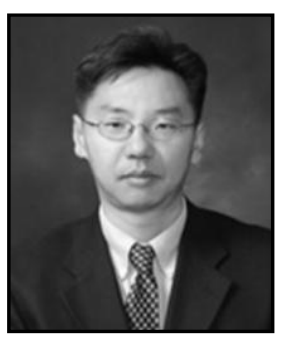

\section{Yoon Sang Kim}

He received the B.S., M.S., and Ph.D. degrees in Electrical Engineering from Sungkyunkwan University, Seoul, Korea, in 1993, 1995, and 1999, respectively. From 1999 to 2000 he was a member of the Postdoctoral Research Staff of Korea Institute of Science and Technology (KIST), Seoul, Korea. From 2000 to 2003 he was a Faculty Research Associate in the Department of Electrical Engineering, University of Washington, Seattle. From 2003 to 2005 he was a Member of the Senior Research Staff, Samsung Advanced Institute of Technology (SAIT), Suwon, Korea. Since 2005, he has been an Associate Professor at the School of Computer Science and Engineering, Korea University of Technology Education (KOREATECH), Cheonan, Korea. His current research interests include Virtual simulation, Power-IT technology, and Bio-informatics. Dr. Kim was awarded the Korea Science and Engineering Foundation (KOSEF) Overseas Postdoctoral Fellow in 2000. $\mathrm{He}$ is a member of IEEE, IEICE, ICASE, KIPS, and KIEE.

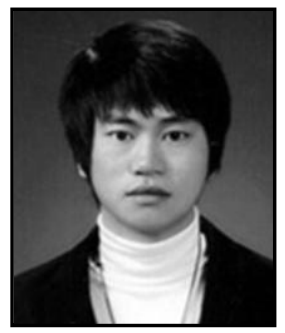

\section{Seong-wook Jang}

He obtained the B.S. degrees in Computer and Science Engineering from Korea University of Technology Education University, Cheonan, Korea, in 2012. he has been a Master's course at the School of Computer and Science Engineering, Korea University of Technology Education (KOREATECH), Cheonan, Korea. His current research interests include Virtual simulation, Bio-informatics.

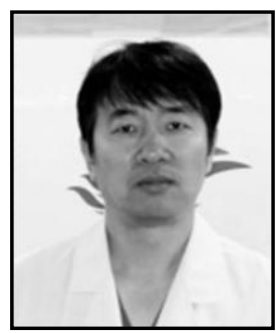

\section{Yon-Sik Yoo}

He obtained the B.S., M.S., and M.D. degrees in from Chungang Medical University, Seoul, Korea, in 1990, 1995, and 1999, respectively. He was a member of the Fellowship of Korea Veterans Hospital Chief of sports department, Pohang St Mary Hospital, Korea. Likewise, he was a Faculty Research Associate in the Department of Biomedical Informatics, University of Pittsburgh, Pittsburgh. He has been a Professor at the Orthopedic department, Hallym Medical University, Dongtan, Korea. His current research interests include Kinematics of knee and shoulder motions, In vivo action of reconstructed ACL and PCL grafts using FEM, and Development of new surgical technique related to shoulder and knee. He is a member of AAOS, ISAKOS, AANA, KOA, and KSES. 